\title{
Supporting Information for: Unexpected anomeric acceptor preference observed using dDNP NMR for transglycosylation studies of $\beta$-galactosidases
}

\author{
Christian Kjeldsen ${ }^{a, b}$, Jan H. Ardenkjær-Larsen ${ }^{b}$, Jens. Ø. Duus*,a
}

${ }^{\mathrm{a}}$ Department of Chemistry and ${ }^{\mathrm{b}} \mathrm{Center}$ for hyperpolarization in magnetic resonance, Department of Health Technology, Technical University of Denmark, 2800 Kgs. Lyngby, Denmark

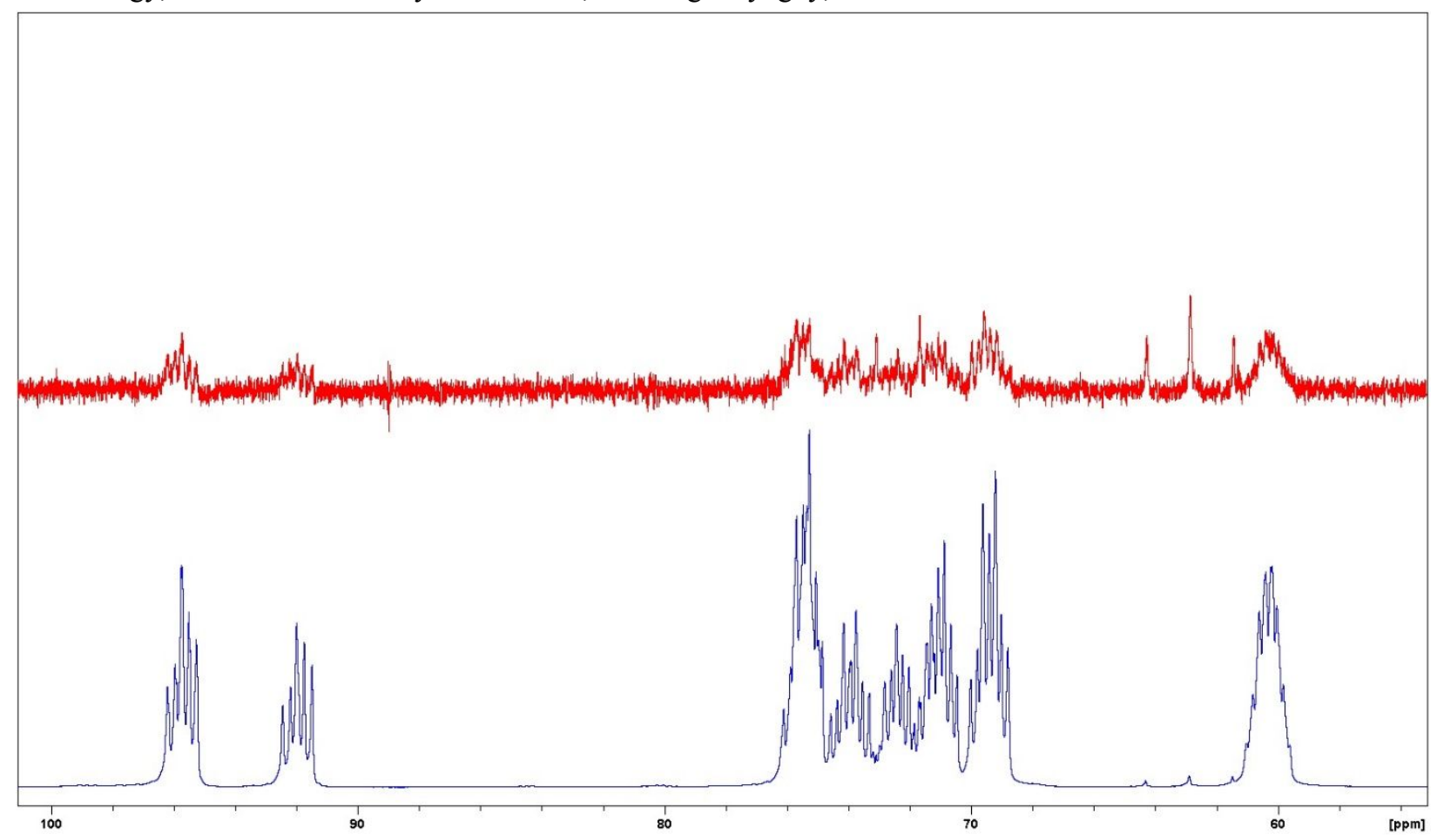

Figure S1. Comparison between first scan after dissolution (blue) and 2048 scans (red) of the same sample. By comparing the SNR of each, the polarization of this particular sample was determined as $25 \%$. For comparison, it would take close to 1 billion scans to achieve the same SNR, which would take upwards of 80 years. The blue spectrum is the same as the first one in figure 3 . The fast relaxing signal at approximately $63 \mathrm{ppm}$ is from the added glycerol used as glassing agent. 


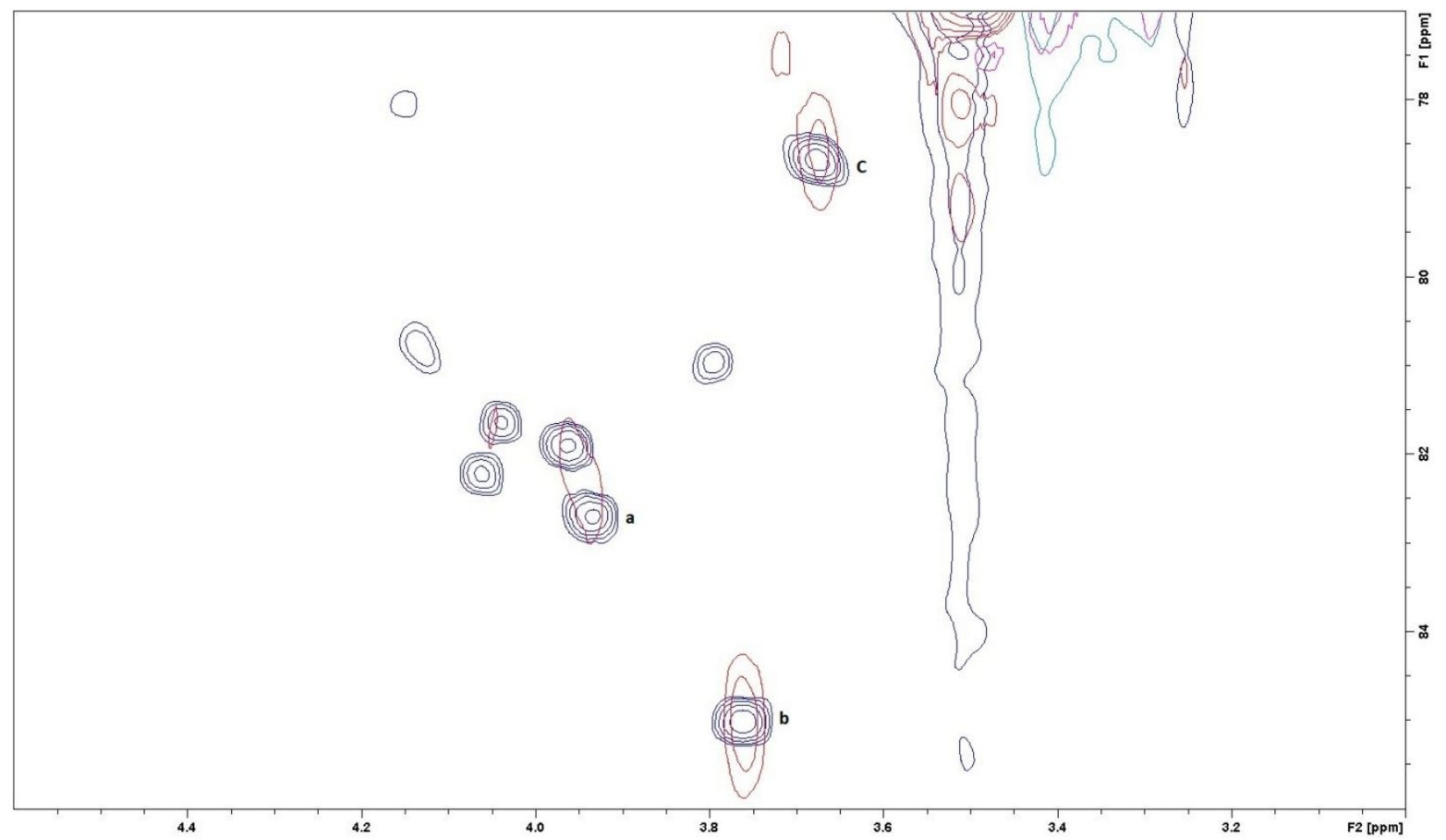

Figure S2. Two overlayed HSQC spectra: Blue: HSQC spectrum of the reaction mixture of Lactozyme 2600L using onp-Galp as donor and glucose as added acceptor, after being quenched at approximately $70 \%$ conversion of the onp-Galp. Red: HSQC spectrum recorded from 2-8 minutes after adding the enzyme of a reaction run under the same conditions. The approximate ratio between 3-substituted $\beta$-glucopyranoside and 3-substituted $\alpha$-glucopyranoside is $8: 2$, whereas at equilibrium (as shown in blue) it is 58:42. The peak labelled as a corresponds to the 3-position of 3-substituted $\alpha$-glc $p$, b to the 3-position of 3-substituted $\beta$-Glc $p$ and $\mathbf{c}$ to the 4-position of both 3-substituted $\alpha$ - and $\beta$-Glc $p$. The additional signals around $\mathbf{a}$ is likely from various transglycosylations to galactose or to onp-Galp, both of which also acts as acceptor. 


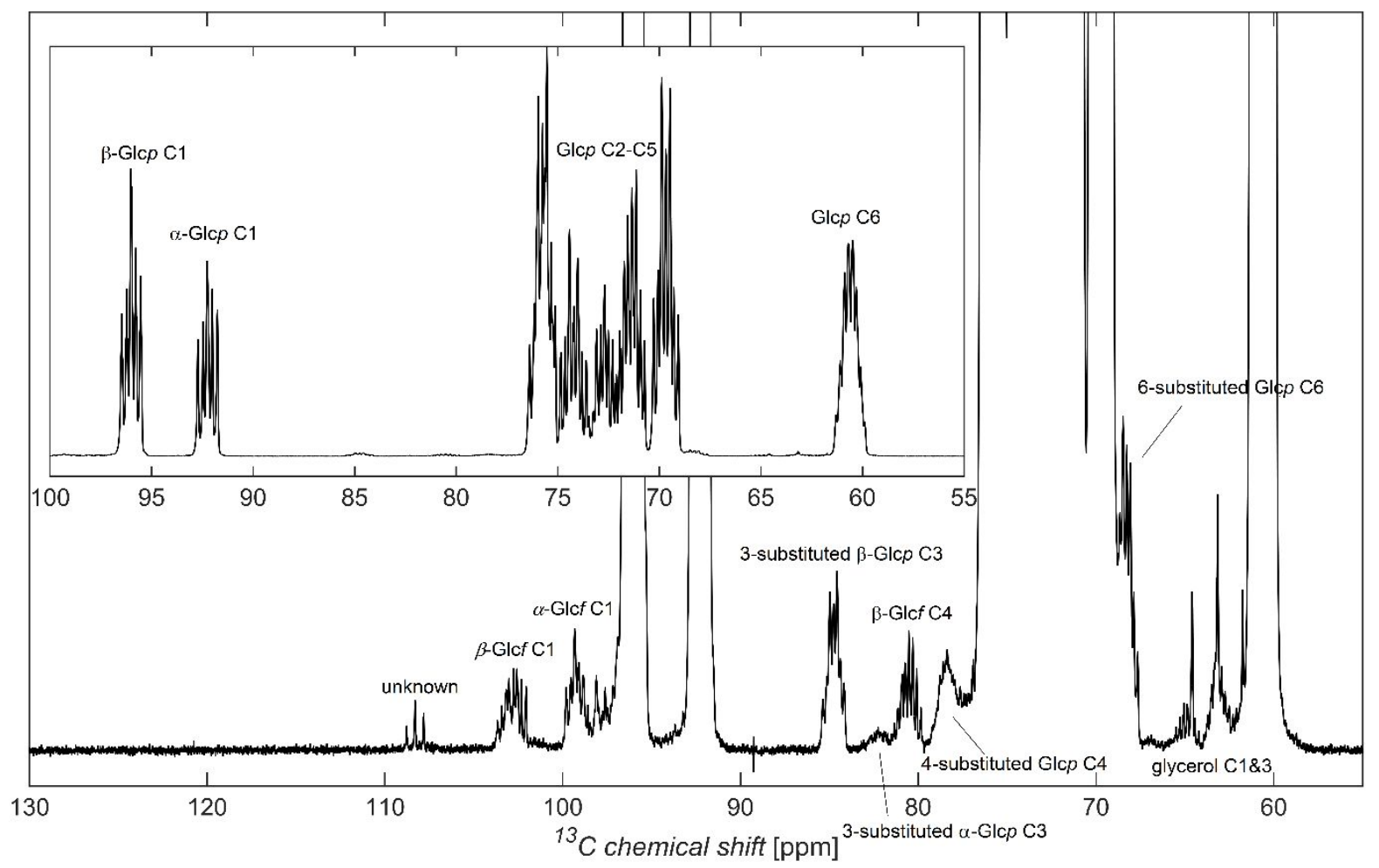

Figure S3. Sums of spectra 3-10 of Hyperpolarized [U-13 C;U-2 H]glucose mixed with onp-Gal $p$ and Lactozyme 2600L with assignments of all distinguishable peaks (corresponds to the middle spectrum is figure 2). The insert is the same spectrum, but zoomed to better visualize the glucopyranose signals. Note that most signals from the transglycosylation products will be overlapping with the much more intense signals from free glucose, and similarly will most of the glucofuranose signals. The signal marked as unknown is also present in the spectrum with only glucose, and arises either from the glucose itself, an impurity in the glucose or from some other part of the DNP solution.

Table S1: ${ }^{13} \mathrm{C}$ assignments of the glucose in each of the disaccharides produced by transglycosylation as well as of free glucose. The bold numbers are to highlight substituted positions.

\begin{tabular}{|l|r|r|r|r|r|r|}
\hline Glucose species & C1 ppm & \multicolumn{1}{c|}{ C2 ppm } & C3 ppm & C4 ppm & C5 ppm & C6 ppm \\
\hline$\alpha$-Glucose & 91.8 & 71.2 & 72.5 & 69.4 & 71.2 & 60.3 \\
\hline$\beta$-Glucose & 95.7 & 73.9 & 75.5 & 69.3 & 75.7 & 60.5 \\
\hline$\alpha$-Allolactose & 91.8 & 71.2 & 72.4 & 69.3 & 70.5 & $\mathbf{6 8 . 4}$ \\
\hline$\beta$-Allolactose & 95.7 & 73.6 & 74.5 & 69.2 & 75.1 & $\mathbf{6 8 . 4}$ \\
\hline$\alpha$-Lactose & 91.8 & 71.1 & 71.4 & $\mathbf{7 8 . 4}$ & 70.1 & 60.1 \\
\hline$\beta$-Lactose & 95.7 & 73.8 & 74.4 & $\mathbf{7 8 . 3}$ & 74.8 & 59.9 \\
\hline$\beta$-Galp-1,3- $\alpha$-Glcp & 91.7 & 70.8 & $\mathbf{8 2 . 3}$ & 69.1 & 71.1 & 60.1 \\
\hline$\beta$-Galp-1,3- $\beta$-Glcp & 95.6 & 73.5 & $\mathbf{8 4 . 7}$ & 69.0 & 75.6 & 59.9 \\
\hline
\end{tabular}

